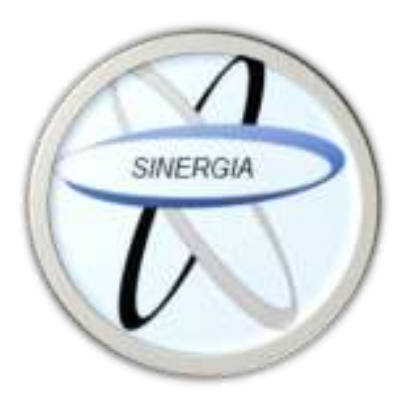

Volumen 4 Número 7

PUBLICACION MENSUAL

Artículo de reporte de casos clínicos:

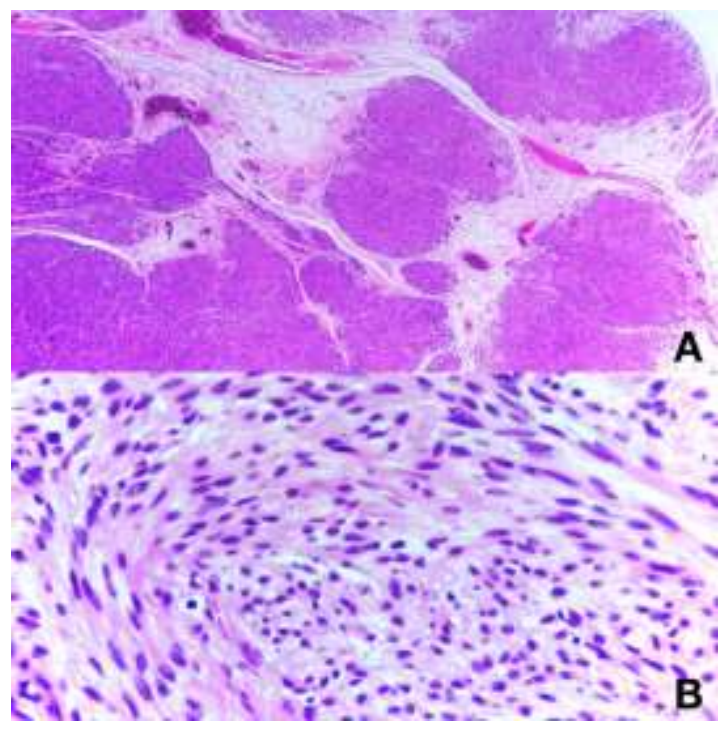

\section{Reporte de caso clínico de leiomiomatosis peritoneal diseminada}

\author{
Disseminated peritoneal \\ leiomyomatosis, case report
}

Autores:

Dra. Ana María Gutiérrez Gallardo

Dra. María Eugenia Mesalles Ramírez
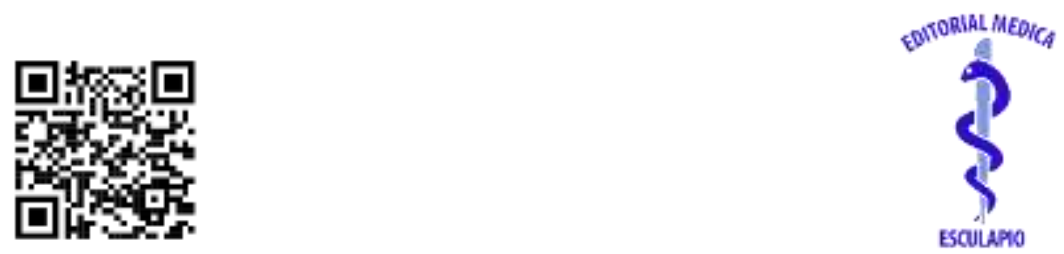

Indexada en:

lattindeX 


\section{CUERPO EDITORIAL}

\section{DIRECTORA}

- Dra. Margarita Karol Malpartida Ampudia, Médico independiente, San José, Costa Rica.

\section{EDITOR}

- Dr. Esteban Sánchez Gaitán, Caja costarricense del Seguro Social, Limón, Costa Rica.

\section{CONSEJO EDITORIAL}

- Dr. Cesar Vallejos Pasache, Hospital III lquitos, Loreto, Perú.

- Dra. Anais López, Hospital Nacional Edgardo Rebagliati Martins, Lima, Perú.

- Dra. Ingrid Ballesteros Ordoñez, Pontificia Universidad Javeriana, Bogotá, Colombia.

- Dra. Mariela Burga, Hospital Nacional Edgardo Rebagliati Martins. Lima, Perú.

- Dra. Patricia Santos Carlín, Ministerio de Salud (MINSA). Lima, Perú.

- Dr. Raydel Pérez Castillo, Centro Provincial de Medicina Deportiva Las Tunas, Cuba.

\section{COMITÉ CIENTÍFICO}

- Dr. Zulema Berrios Fuentes, Ministerio de Salud (MINSA), Lima, Perú.

- Dr. Gerardo Francisco Javier Rivera Silva, Universidad de Monterrey, Nuevo León, México.

- Dr. Gilberto Malpartida Toribio, Hospital de la Solidaridad, Lima, Perú.

- Dra. Marcela Fernández Brenes, Caja costarricense del Seguro Social, Limón, Costa Rica

- Dr. Hans Reyes Garay, Eastern Maine Medical Center, Maine, United States.

- Dr. Steven Acevedo Naranjo, Saint- Luc Hospital, Quebec, Canadá.

- Dr. Luis Osvaldo Farington Reyes, Hospital regional universitario Jose Maria Cabral y Baez, Republica Dominicana.

- Dra. Caridad Maria Tamayo Reus, Hospital Pediátrico Sur Antonio María Béguez César de Santiago de Cuba, Cuba.

- Dr. Luis Malpartida Toribio, Hospital Nacional Daniel Alcides Carrión, Callao, Perú.

- Dra. Allison Viviana Segura Cotrino, Médico Jurídico en Prestadora de Salud, Colombia.

\section{EQUÍPO TÉCNICO}

- Msc. Meylin Yamile Fernández Reyes, Universidad de Valencia, España.

- Lic. Margarita Ampudia Matos, Hospital de Emergencias Grau, Lima, Perú.

- Ing. Jorge Malpartida Toribio, Telefónica del Perú, Lima, Perú.

- Srta. Maricielo Ampudia Gutiérrez, George Mason University, Virginia, Estados Unidos.

\section{EDITORIAL ESCULAPIO}

50 metros norte de UCIMED, Sabana Sur, San José-Costa Rica Teléfono: 8668002

E-mail:

revistamedicasinergia@gmail.com

\section{ENTIDAD EDITORA}

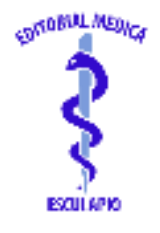

Rica

\section{SOMEA}

SOCIEDAD DE MEDICOS DE AMERICA

Frente de la parada de buses Guácimo, Limón. Costa

Teléfono: 8668002

Sociedaddemedicosdeamerica@hotmail.com

https://somea.businesscatalyst.com/informacion.html 


\section{Reporte de caso clínico de leiomiomatosis peritoneal diseminada}

Disseminated peritoneal leiomyomatosis, case report

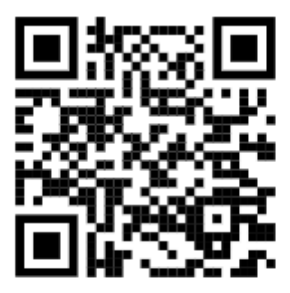

RECIBIDO

$29 / 04 / 2019$

\author{
${ }^{1}$ Dra. Ana María Gutiérrez Gallardo \\ Hospital San Juan de Dios, San José, Costa Rica \\ (D) https://orcid.org/0000-0003-0711-8450
}

${ }^{2}$ Dra. María Eugenia Mesalles Ramírez Investigadora independiente, San José, Costa Rica

https://orcid.org/0000-0002-3190-3371
${ }^{1}$ Residente de ginecología

y obstetricia

CodMED14462

anigu10@hotmail.com

${ }^{2}$ Médica general, graduada

de la Universidad de

Ciencias Médicas

(UCIMED).

CodMED16130

mariae.mesalles@gmail.com

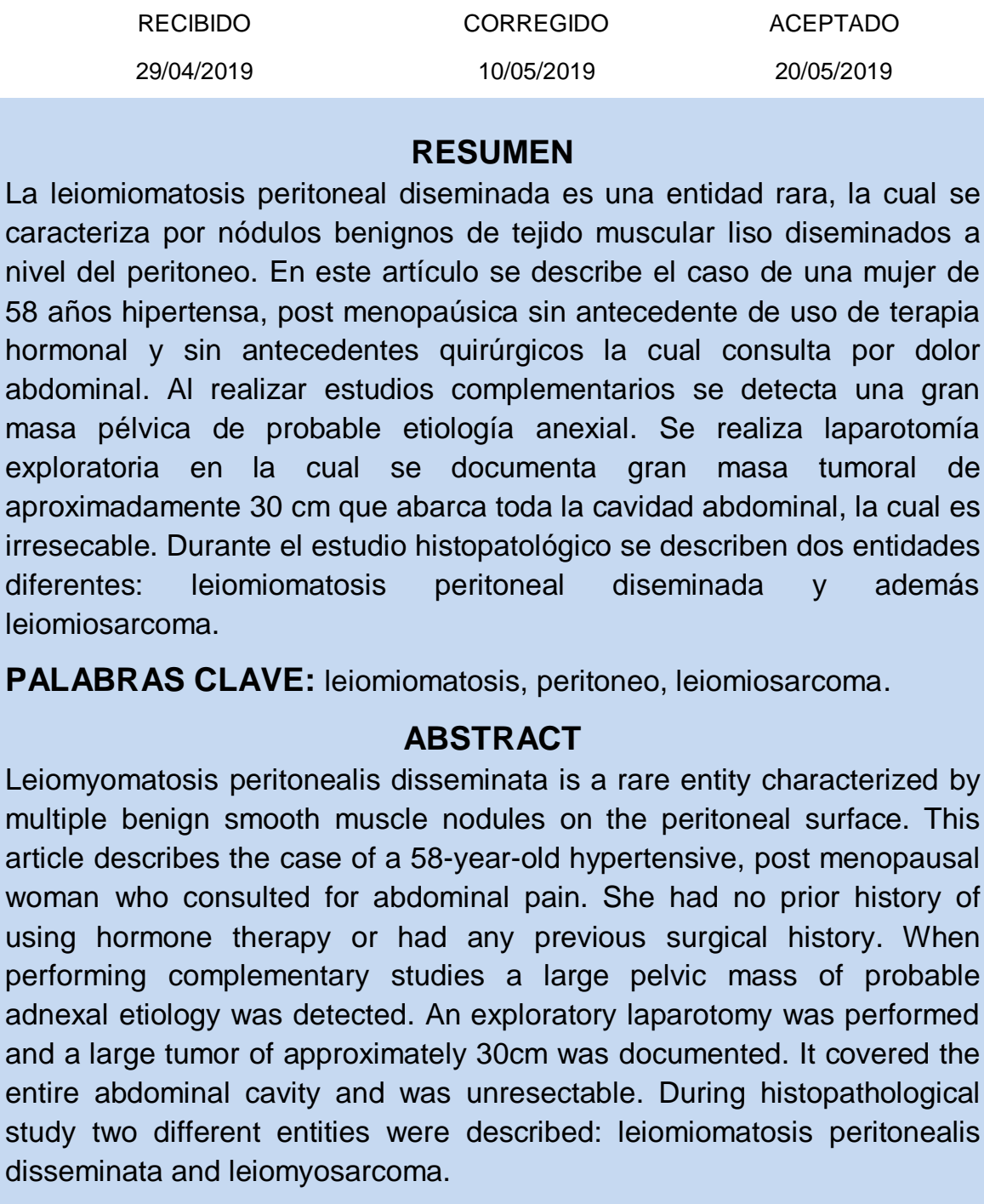


KEYWORDS: leiomyomatosis, peritoneum, leiomyosarcoma.

\section{INTRODUCCIÓN}

Leiomiomatosis peritoneal diseminada (LPD) es una enfermedad rara, benigna, que se caracteriza por la presencia de lesiones nodulares de músculo liso que se adhieren o invaden superficialmente el peritoneo. Se describió por primera vez en 1952 por Wilson y Peale y en 1965 Taubert et al. la identificó como una identidad patológica $(1,2)$. La etiología no está clara, sin embargo se han propuesto múltiples hipótesis sobre su fisiopatología (3). Se cree que existe importante subdiagnóstico de esta condición, habiendo limitados casos reportados en la literatura, debido a que usualmente se trata de una entidad asintomática (3). El siguiente artículo presenta una revisión bibliográfica y el caso de una paciente con diagnótico de leiomiomatosis peritoneal diseminada documentado en Costa Rica.

\section{PRESENTACIÓN DE CASO}

Femenina 58 años, hipertensa crónica, sin antecedentes quirúrgicos, núbil, postmenopaúsica, sin antecedentes de uso de terapia de remplazo hormonal. Fue referida por dolor abdominal inespecífico de 2 días de evolución. Se realiza ultrasonido de abdomen en el servicio de emergencias en el que se reporta masa de al menos $215 \times 124 \times$ $175 \mathrm{~mm}$ con volumen de aproximadamente $2500 \mathrm{cc}$, hetereogénea, mal delimitada con componente quístico y sólido; las áreas sólidas presentaban vascularidad al Doppler. La masa se extendía hasta el epigastrio y flanco izquierdo. Los ovarios no se observaban. Además, llamaba la atención no poder definir contornos uterinos por lo que impresionaba masa compleja de origen uterino versus anexial, sin poder descartar otras patologías. Se da el diagnóstico ecográfico de gran masa abdominal de tipo mixto (sólido - quístico) a complementar con estudios de extensión. Debido a estos hallazgos, se realiza tomografía computarizada de abdomen y pelvis con doble medio de contraste en el que se documenta a nivel pélvico, una formación tumoral gigante heterogénea con componente sólido y quístico que se ubica en la parte central de la excavación pélvica y que asciende hacia cavidad abdominal. Es de contorno lobulado con áreas quísticas, las cuales presentan atenuación de 10-15 UH sugiriendo líquido grumoso y parte sólida con atenuación media de 40-50 UH, midiendo $268 \times 125 \times 292 \mathrm{~mm}$ en sagital, AP y transversal, con volumen de 5000 cc. Dicha formación comprimía extrínsecamente la vejiga en sentido inferior y el útero atrófico en sentido posterior. Las asas de intestino delgado con contraste eran desplazadas en sentido superior por la formación. El aspecto y configuración de la masa sugerían proceso tumoral solido quístico a definir (Ver FIGURA 1 Y 2). En el estudio se sugería que podía corresponder con masa anexial, sin embargo por ubicación de la masa, era difícil definir de qué anexo se originaba. No se veía compromiso por ascitis, ganglios retroperitoneales, hidronefrosis, nódulos hepáticos ni derrame pleural. 
La impresión diagnóstica de la tomografía fue: formación tumoral sólido quística de bordes lobulados que se ubica en parte central de excavación pélvica y asciende a cavidad abdominal. De naturaleza a definir.

FIGURA 1. Corte sagital de TAC de paciente, leer descripción en texto. Cortesía servicio de Radiología Hospital San Juan de Dios

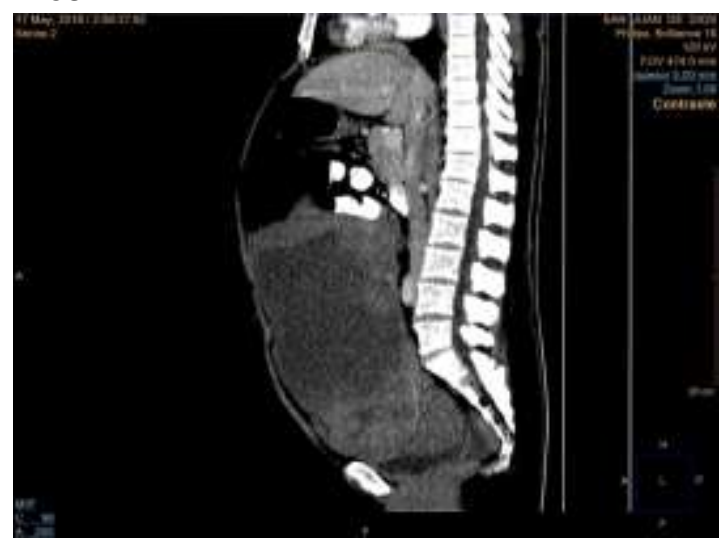

FIGURA 2. Corte coronal de TAC de paciente, leer descripción en texto. Cortesía servicio de Radiología Hospital San Juan de Dios.

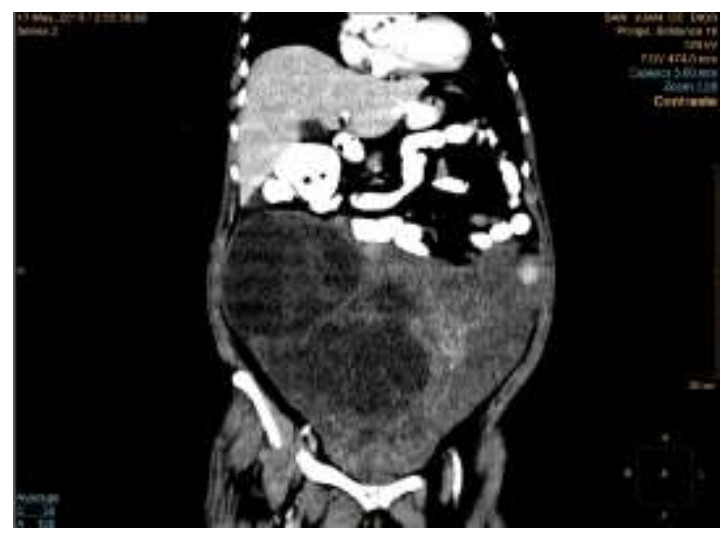

Se realizaron laboratorios de los cuales las únicas alteraciones fueron leucocitosis sin foco infeccioso y el único marcador tumoral elevado fue el CA 125. Debido a que hallazgos de la tomografía y el ultrasonido sugerían masa de origen anexial, es trasladada a ginecología donde se ingresó para laparotomía exploratoria por masa pélvica de posible origen anexial. Durante la laparotomía se documenta presencia de masa tumoral de aproximadamente $30 \mathrm{~cm}$ que abarca toda la cavidad abdominal con excrecencias papilares a lo largo de toda la masa, la cual es irresecable

Se realizó una biopsia inicial en la que se documentó en todos los cortes histológicos examinados fragmentos de tejido conectivo con nodulaciones compuestas de células fusiformes que se disponen en haces entrecruzados sin observarse en ninguno de ellos presencia de datos de malignidad. (Ver FIGURA 3).

FIGURA 3. A) Aumento a $4 X$, fragmentos de tejido conectivo con nodulaciones de músculo liso, bien delimitadas. B) Aumento a 40X, células fusiformes en haces entrecruzados que no presentan datos de malignidad. Cortesía servicio de Patología, Hospital San Juan de Dios.

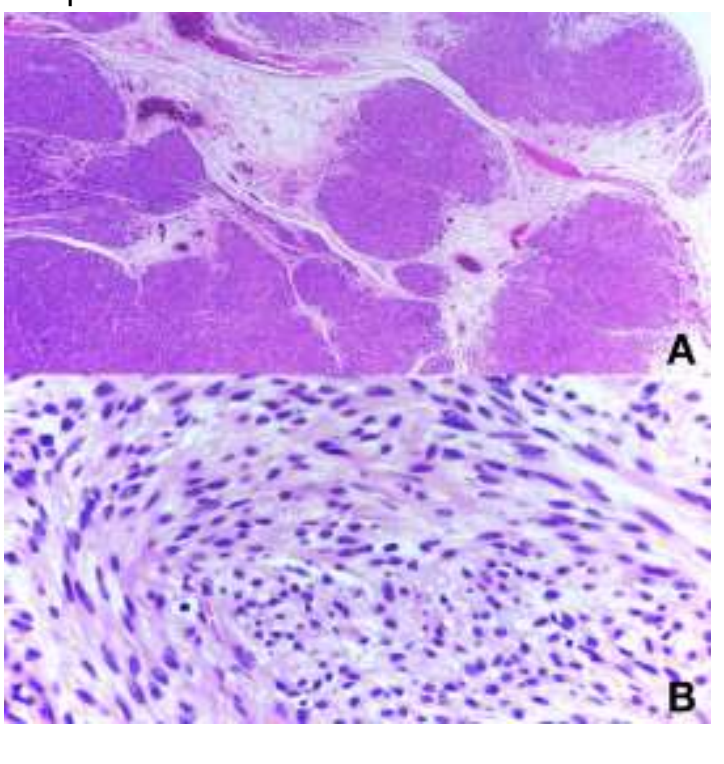

Se realizaron tinciones de inmunohistoquímica siendo receptores de estrógeno, progesterona, desmina y 
actina músculo liso positivas y con un KI67 bajo. Sin embargo, por los hallazgos radiológicos se decide tomar una nueva biopsia en la que se evidencia nuevamente estos nódulos musculares benignos y en uno de ellos se observa una zona de transición con mitosis, necrosis y atipia celular, por lo que se documenta un leiomiosarcoma de alto grado concomitante (Ver FIGURA 4). Por lo que el diagnóstico patológico final fue leiomiomatosis peritoneal diseminada con leiomiosarcoma concomitante.

FIGURA 4. Zona de necrosis y hemorragia donde se observan escasas mitosis. Cortesía servicio de Patología Hospital San Juan de Dios

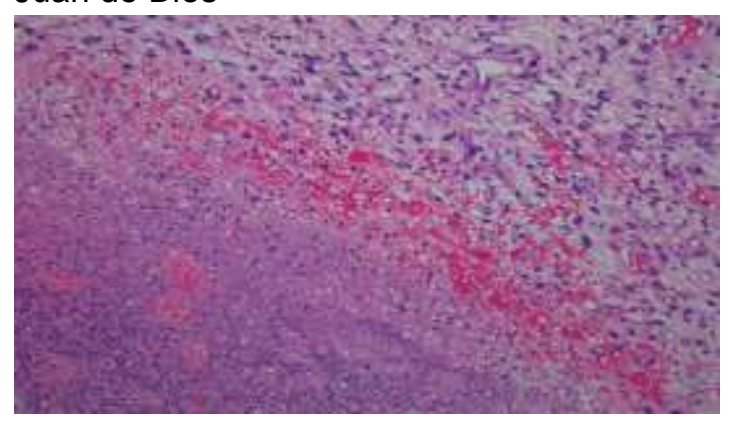

\section{DISCUSIÓN Y REVISIÓN DE LA LITERATURA MÉDICA}

\section{DEFINICIÓN Y EPIDEMIOLOGÍA}

La LPD es una condición poco común y de naturaleza benigna. Esta entidad se caracteriza por múltiples nódulos peritoneales 0 subperitoneales constituidos por células de músculo liso. Dichos nódulos varían de tamaño desde milímetros hasta centímetros y pueden ser similares a una carcinomatosis peritoneal $(3,4)$. Además, estos nódulos pueden ser mixtos y tener componente tanto quístico como sólido, e incluso pueden ser hemorrágicos (5).

Es una entidad que en la mayoría de pacientes es asintomática y por lo tanto se cree que hay un alto subdiagnóstico (3). Se estipula entonces que su incidencia exacta es desconocida, pero hay aproximadamente 193 casos reportados en la literatura según artículo publicado en 2017 (5).

\section{FACTORES DE RIESGO Y ETIOLOGÍA}

La etiología de esta entidad es aún poco clara (3). Sin embargo, se han planteado diversas hipótesis sobre la fisiopatología de esta condición. Dichas causas se pueden dividir en hormonales, metaplasia de células madre mesenquimales subperitoneales, genéticas o iatrogénicas posteriores a morcelación de mioma durante cirugía laparoscópica (4).

Se ha postulado que puede existir una predisposición o sensibilidad selectiva, poco común, que lleva a metaplasia de las células madre mesenquimales. Se cree que este proceso se da por estimulación hormonal, promoviendo la diferenciación de estas células a mioblastos, miofibroblastos, fibroblastos e incluso a células similares a la decidua. Esta hipótesis se respalda en el hecho de que se ha encontrado esta entidad en pacientes embarazadas, posterior a uso prolongado de anticonceptivos orales, terapia de reemplazo hormonal, tamoxifeno, en pacientes con tumores secretores de estrógeno (tumores de células de la granulosa) o posterior a estimulación ovárica $(4,6)$.

Otro hallazgo que sugiere rol hormonal 
en la patogénesis de la LPD, es que se han encontrado receptores de estrógeno y progesterona en estas células tumorales (3). Otro dato sugestivo, es que se puede lograr regresión de esta entidad con la disminución de niveles de estrógeno con agonistas de la hormona liberadora de gonadotropina, inhibidores de aromatasa 0 al suspender anticonceptivos orales y terapia de reemplazo hormonal (4).

Sin embargo, también se ha encontrado esta entidad en paciente que no han sido expuestas a estrógenos o progestinas exógenas (4). A pesar que es una condición clínica que afecta principalmente a mujeres premenopáusicas, también hay casos reportados en mujeres postmenopáusicas y hombres sin exceso de hormonas identificable. Esto podría deberse en parte a un aumento en la respuesta de células tumorales a niveles hormonales normales. De hecho, se ha detectado un aumento de receptores de estrógeno y progesterona en estas células al realizar ensayos inmunohistoquímicos (6).

En cuanto a la teoría genética, se ha sugerido que alguna anormalidad en cromosoma $\mathrm{X}$ y otros cromosomas incluyendo el 8, 12 y 17 podrían estar involucrados en una patogénesis en común entre miomas uterinos y LPD. Las células que proliferan y se implantan en peritoneo podrían ser originarias de un mioma uterino (4). Sin embargo debido a que esta entidad se ha presentado en hombres y se ha identificado un grupo familiar con esta condición, se sugiere que es una nueva condición autosómica dominante con niveles variables de penetrancia $(4,7)$.
Por otro lado, se ha planteado una teoría iatrogénica como etiología de esta entidad. Se sugiere la posibilidad de que pequeños fragmentos desprendidos de mioma en una miomectomía laparoscópica por morcelación, pueden implantarse en el peritoneo, crecer, diseminarse a través del trayecto laparoscópico y convertirse en LPD $(3,4,6)$. Esta hipótesis surge debido a que no se ha reportado casos posteriores a miomectomía o histerectomía abdominal, y a que hay un aumento de casos de LPD posterior a miomectomía laparoscópica cuando se usa morcelación para lograr extracción del mioma por esta vía. Además se han encontrado nódulos de LPD alrededor de la cicatriz de laparoscopía de estos casos (4).

La hipótesis de que la etiología puede deberse a metástasis de una enfermedad unicéntrica, más comúnmente leiomiomatosis uterina, se sustenta en el origen monoclonal de cada uno de estos tumores y a la correlación de hallazgos en citogenética molecular entre los leiomiomas uterinos y la LPD en pacientes con historia de laparoscopía con morcelación de leiomioma (8).

\section{CLÍNICA, DIAGNÓSTICO Y DIAGNOSTICOS DIFERENCIALES}

En cuanto a la presentación clínica, en la mayoría de casos se trata de una condición asintomática o un hallazgo incidental $(9,10)$. Dentro de los posibles síntomas en estas pacientes se encuentran, problemas abdominales inespecíficos como dolor, distensión o malestar abdominal, menstruación irregular o abundante o sensación de 
masa en abdomen inferior. En raras ocasiones puede presentarse con síntomas de obstrucción abdominal por masa abdominal, sangrado gastrointestinal e incluso abdomen agudo debido a erosiones de LPD en intestinos (11).

La detección de los nódulos abdominales durante los estudios diagnósticos de imágenes frecuentemente es incidental, suele ser un hallazgo radiológico o quirúrgico. Se puede sospechar el diagnóstico con imágenes, que en algunas circunstancias puede mostrar nódulos a nivel peritoneal. Se han utilizado el ultrasonido abdominal, tomografía axial computarizada y la resonancia magnética como estudios de gabinete $(9,12)$. Sin embargo, los hallazgos se pueden confundir fácilmente con carcinomatosos peritoneal, la cual más comúnmente se asocia con otros síntomas como pérdida de peso, ascitis y enfermedad progresiva observada en imágenes $(6,13)$. Solamente el diagnóstico histopatológico permite diferenciar LPD de sus diagnósticos diferenciales dentro de los cuales se encuentran: metástasis peritoneales diseminadas, leiomiosarcomas del útero y leiomioma benigno metastásico $(3,9)$.

\section{PATOLOGÍA}

Microscópicamente, LPD consiste de células fusiformes, dispuestas en fascículos entrelazados más celulares que el miometrio normal. Puede haber degeneración quística con contenido líquido seroso o calcificación. Los nódulos carecen de figuras mitóticas o tienen índices mitóticos menores a 3 mitosis por 10 campos de alto poder. También carecen de atipia celular, pleomorfismo nuclear, hipercromasía y necrosis. Todos estos elementos en conjunto con un patrón de crecimiento infiltrativo son componentes sugestivos de una neoplasia de mayor grado y distinta denominada leiomiosarcoma. El estudio inmunohistoquímico es positivo para vimentina, desmina, actina de músculo liso y actina de músculo especifico (14). Estos tumores demuestran en la inmunohistoquímica la presencia de receptores hormonales estrogénicos y de progesterona, siendo hormono respondedores en su gran mayoría (15).

LPD se considera una enfermedad benigna, pero en $2-5 \%$ de los casos reportados se reporta progresión a malignidad $(12,16,17)$.

\section{TRATAMIENTO}

El tratamiento se establece según la edad, el estado hormonal, reproductivo y la sintomatología del paciente. En pacientes asintomáticas se puede vigilar con ultrasonido. Si hay sospecha de malignización 0 las lesiones son recurrentes, la indicación es resección de los nódulos más salpingo-ooforectomía, histerectomía y linfadenectomía (15). También se ha utilizado tratamiento hormonal para suprimir el crecimiento de las lesiones (18).

El tratamiento médico hormonal es efectivo y pueden utilizarse los inhibidores de aromatasa o agonistas de hormona liberadora de gonadotropina. Por otro lado, la cirugía extensa debe considerarse cuando existe compresión de órganos, como en casos donde se presenta con obstrucción intestinal (19). Además, una importante intervención no 
quirúrgica es limitar o descontinuar la exposición hormonal (anticonceptivos orales o terapia de remplazo hormonal). Dicha intervención ha demostrado disminuir el tamaño de estos tumores $(5,20)$.

\section{CONCLUSIÓN}

La LPD es una enfermedad benigna que puede semejar una carcinomatosis peritoneal o leiomiosarcoma metastásico. Su diagnóstico diferencial es difícil, requiere de una buena historia clínica, resección quirúrgica y análisis patológico. Hay que tener gran cautela al diagnosticarla, ya que en algunos casos como en el presentado, puede haber degeneración maligna concomitante. Es fundamental que los médicos conozcan de esta rara entidad para poder dar el diagnóstico y el tratamiento apropiado.

\section{AGRADECIMIENTOS}

Se le agradece profundamente a la Dra. Sylvia Molina Mainieri y al Dr. Pablo Goyenaga Castro, ambos patólogos del Hospital San Juan de Dios, por la ayuda brindada en el desarrollo de este artículo.

\section{REFERENCIAS}

1. Willson JR, Peale AR. Multiple Peritoneal Leiomyomas Associated with a Granulosa-Cell Tumor of the Ovary Presented at a meeting of the Philadelphia Obstetrical Society, Jan. 3, 1952.. American Journal of Obstetrics and Gynecology. 1952 07;64(1):204-208. https://doi.org/10.1016/s0002-9378(16)38757-9

2. Taubert HD, Wissner SE, Haskins AL. Leiomyomatosis Peritonealis Disseminata; An Unusual Complication Of Genital Leiomyomata. Obstet Gynecol. 1965;25(4):561-74.

3. Psathas G, Zarokosta M, Zoulamoglou M, Chrysikos D, Thivaios I, Kaklamanos I, Birbas K, Mariolis-Sapsakos T. Leiomyomatosis peritonealis disseminata: A case report and meticulous review of the literature. International Journal of Surgery Case Reports. 2017;40:105-

108. https://doi.org/10.1016/j.ijscr.2017.09.016

4. Al-Talib A, Tulandi T. Pathophysiology and Possible latrogenic Cause of Leiomyomatosis Peritonealis Disseminata. Gynecologic and Obstetric Investigation. 2010;69(4):239-

244. https://doi.org/10.1159/000274487

5. Gaichies L, Fabre-Monplaisir L, Fauvet R, Alves A, Mulliri A. Leiomyomatosis peritonealisis disseminata: Two unusual cases with literature review. Journal of Gynecology Obstetrics and Human Reproduction. 2018 02;47(2):89-94. https://doi.org/10.1016/j.jogoh.2017.11.011

6. Fasih N, Prasad Shanbhogue AK, Macdonald DB, Fraser-Hill MA, Papadatos D, Kielar AZ, Doherty GP, Walsh C, Mclnnes M, Atri M. Leiomyomas beyond the Uterus: Unusual Locations, Rare Manifestations. RadioGraphics. 2008 Nov;28(7):1931-1948. https://doi.org/10.1148/rg.287085095

7. Halama N, Grauling-Halama SA, Daboul I. Familial clustering of Leiomyomatosis peritonealis disseminata: an unknown genetic syndrome?. BMC Gastroenterology. 2005 Oct 13;5(1). https://doi.org/10.1186/1471 $\underline{230 x-5-33}$

8. Ordulu Z, Cin PD, Chong WW, Choy KW, Lee C, Muto MG, Quade BJ, Morton CC. Disseminated peritoneal leiomyomatosis after laparoscopic supracervical hysterectomy with characteristic molecular cytogenetic findings of uterine leiomyoma. Genes, Chromosomes and Cancer. 201009 14;49(12):11521160. https://doi.org/10.1002/gcc.20824 
9. Bekkers R, Willemsen W, Schijf C, Massuger L, Bulten J, Merkus J. Leiomyomatosis Peritonealis Disseminata: Does Malignant Transformation Occur? A Literature Review. Gynecologic Oncology. 1999 Oct;75(1):158-163. https://doi.org/10.1006/gyno.1999.5490

10. Ernesto L, Valencia G, Rodrigo R, Merino A, José J, Fuentes G, et al. Leiomiomatosis peritoneal diseminada en una paciente con endometriosis. 2014;(3):131-4.

11. Nappi L, Sorrentino F, Angioni S, Pontis A, Barone I, Greco P. Leiomyomatosis Peritonealis Disseminata (LPD) ten years after laparoscopic myomectomy associated with ascites and lymph nodes enlargement: acase report. International Journal of Surgery Case Reports. 2016;25:13. https://doi.org/10.1016/j.ijscr.2016.05.017

12. Abulafia O, Angel C, Sherer DM, Fultz PJ, Bonfiglio TA, DuBeshter B. Computed tomography of leiomyomatosis peritonealis disseminata with malignant transformation. American Journal of Obstetrics and Gynecology. 1993 07;169(1):52-54. https://doi.org/10.1016/0002-9378(93)90130-b

13. Montero D JC, Iturain M N, Urrea B D, Misad S C. LEIOMIOMATOSIS PERITONEAL DISEMINADA Y EMBARAZO. Revista chilena de obstetricia y ginecología. 2008;73(2). https://doi.org/10.4067/s0717$\underline{75262008000200011}$

14. Al-Talib A, Al-Farsi AR, Stanimir G. Leiomyomatosis peritonealis disseminata with features of carcinomatosis on laparoscopy a case report. Vol. 9, Sultan Qaboos University Medical Journal. 2009. p.315-8.

15. Benítez Cáceres H, Van Der Linde Rosemberg V, Parra Lara R. Leiomiomatosis Peritoneal Diseminada. International Journal of Morphology. 2014 06;32(2):666 670. https://doi.org/10.4067/s0717$\underline{95022014000200047}$

16. Lamarca $M$ Rubio $P$ Andrés $P$ Rodrigo $C$ Leiomyomatosis peritonealis disseminata with malignant degeneration. Eur J Gynaecol Oncol. 2011;32(6):702-4.

17. Shahin NA, Al-Khader A, Ahram M, Elmuhtaseb MS, Bata MS. Case Report Malignant leiomyomatosis peritonei display inter-tumor genetic heterogeneity among synchronous and metachronous lesions: molecular evidence from a single case. Am J Clin Exp Obs Gynecol. 2017;4(2):29-36. http://ajceog.us/files/ajceog0049045.pdf

18. Hales HA, Peterson CM, Jones KP, Quinn JD. Leiomyomatosis peritonealis disseminata treated with a gonadotropin-releasing hormone agonist. American Journal of Obstetrics and Gynecology. 199208;167(2):515-516. https://doi.org/10.1016/s0002-9378(11)91445-8

19. Julien C, Bourgouin S, Boudin L, Balandraud P. Disseminated Peritoneal Leiomyomatosis. J Gastrointest Surg. 2018 Jun 29;36(1):57-9.

20. Karuppaswamy J, Tapp A. Leiomyomatosis peritonealis disseminata - Is a different approach needed? $\begin{array}{llllll}\text { Journal of Obstetrics and Gynaecology. } 2002 & 01 ; 22(4): 446 & 447 .\end{array}$ https://doi.org/10.1080/01443610220141498 